Bull. Austral. Math. Soc.

VoL. 56 (1997) [197-208]

\title{
SYSTEMS OF DIFFERENTIAL EQUATIONS WITH FULLY NONLINEAR BOUNDARY CONDITIONS
}

\author{
H.B. THOMPSON
}

We give sufficient conditions involving $f, g$ and $\boldsymbol{\Omega}$ in order that systems of differential equations of the form $y^{\prime \prime}=f\left(x, y, y^{\prime}\right), x$ in $[0,1]$ with fully nonlinear boundary conditions of the form $g\left((y(0), y(1)),\left(y^{\prime}(0), y^{\prime}(1)\right)\right)=0$ have solutions $y$ with $(x, y)$ in $\overline{\boldsymbol{\Omega}} \subseteq[0,1] \times \mathbf{R}^{n}$. We use Schauder degree theory in a novel space. Well known existence results for the Picard, the periodic and the Neumann boundary conditions follow as special cases of our results.

\section{INTRODUCTION}

In this paper we obtain existence results for solutions of second order systems of ordinary differential equations with fully nonlinear boundary conditions which include the Picard, the periodic and Neumann boundary conditions as special cases.

In Section 2 we consider the problem

$$
y^{\prime \prime}=f\left(x, y, y^{\prime}\right) \quad x \in[0,1]
$$

with the fully nonlinear boundary conditions

$$
g\left((y(0), y(1)),\left(y^{\prime}(0), y^{\prime}(1)\right)\right)=0 .
$$

The Picard (also called Dirichlet) boundary conditions correspond to the special case

$$
\begin{aligned}
& g^{0}\left((y(0), y(1)) ;\left(y^{\prime}(0), y^{\prime}(1)\right)\right)=y(0)-A=0 \text { and } \\
& g^{1}\left((y(0), y(1)) ;\left(y^{\prime}(0), y^{\prime}(1)\right)\right)=y(1)-B=0,
\end{aligned}
$$

while the Neumann boundary conditions correspond to the special case

$$
\begin{aligned}
& g^{0}\left((y(0), y(1)) ;\left(y^{\prime}(0), y^{\prime}(1)\right)\right)=y^{\prime}(0)-A=0 \text { and } \\
& g^{1}\left((y(0), y(1)) ;\left(y^{\prime}(0), y^{\prime}(1)\right)\right)=y^{\prime}(1)-B=0,
\end{aligned}
$$

and the periodic boundary conditions correspond to the special case

$$
\begin{aligned}
& g^{0}\left((y(0), y(1)) ;\left(y^{\prime}(0), y^{\prime}(1)\right)\right)=y(0)-y(1)=0 \text { and } \\
& g^{1}\left((y(0), y(1)) ;\left(y^{\prime}(0), y^{\prime}(1)\right)\right)=y^{\prime}(1)-y^{\prime}(0)=0 .
\end{aligned}
$$

Received 3rd October, 1996

Copyright Clearance Centre, Inc. Serial-fee code: 0004-9729/97 \$A2.00+0.00. 
A solution $y$ is a twice continuously differentiable, $\mathbf{R}^{n}$ valued function satisfying (1.1) everywhere and the boundary conditions (1.2). Our proofs are based on Schauder degree theory and ideas introduced by the author in [10]. There is an extensive literature on two point boundary value problems related to the class of problems we consider. Our results extend some of those of Bebernes and Schmitt [1], Habets and Schmitt [5], Gaines and Mawhin [3], Knobloch [6], Knobloch and Schmitt [7], Lan [8], Granas, Guenther and Lee [4], and the author [11]. Gaines and Mawhin [3] were the first to consider second order systems in the present context.

For the convenience of the reader we include the following notation.

For a bounded open subset $T$ of $[0,1] \times \mathbf{R}^{n}$, let $\partial T$ denote the boundary of $T$, let $\bar{T}$ denote its closure and for $x \in[0,1]$ let $T(x)$ denote its $x$-cross section and $\partial T(x)$ denote the boundary of $T(x)$. Thus $T(x)=\left\{y \in \mathbf{R}^{n}:(x, y) \in T\right\}$. By the boundary of $T$ we mean the curved boundary so that we exclude the sets $\{0\} \times T(0)$ and $\{1\} \times T(1)$. Let $y=\left(y_{1}, \cdots, y_{n}\right) \in \mathbf{R}^{n}, y^{T}$ denote the transpose of $y, I$ denote the identity on $\mathbf{R}^{n}$ so $I(y)=y$ for all $y, I^{-}$denote the mapping given by $I^{-}(y)=\left(y_{1},-y_{2}, \ldots,-y_{n}\right)$ for all $y, B_{r}=\left\{y \in \mathbf{R}^{n}:|y|<r\right\}$, and $B(x, r)=\left\{y \in \mathbf{R}^{n}:|y-x|<r\right\}$. Also if $A$ and $B$ are subsets of $\mathrm{R}^{n}$ we denote by $C^{m}(A, B)$ the set of functions from $A$ to $B$ with continuous $m$-th order partial derivatives endowed with the usual maximum norm. If $A$ is a bounded open subset of $\mathbf{R}^{n}, p \in \mathbf{R}^{n}, \mathcal{G} \in C\left(\bar{A} ; \mathbf{R}^{n}\right)$ and $p \notin \mathcal{G}(\partial A)$ we denote the Brouwer degree of $\mathcal{G}$ on $A$ at $p$ by $a^{3}(\mathcal{G}, A, p)$.

Let $\boldsymbol{\Omega}$ be a bounded open subset of $[0,1] \times \mathbf{R}^{n}$. If $0 \in \Omega(x)$ for all $x \in[0,1]$, then we define $L:[0,1] \times \mathbf{R}^{n} \rightarrow \mathbf{R}$ by

$$
L(x, y)= \begin{cases}1, & \text { for }(x, y) \in \overline{\boldsymbol{\Omega}} \\ \inf \{k>0:(x, y / k) \in \boldsymbol{\Omega}\}, & \text { otherwise. }\end{cases}
$$

Thus $L \geqslant 1$ and $(x, y / L(x, y))$ belongs to the boundary of $\Omega$ for all $(x, y)$ not in $\boldsymbol{\Omega}$. When $y$ is a function of $x$ uniquely determined from the context we shall abbreviate $L(x, y(x))$ to $L(x)$.

\section{NONLINEAR BOUNDARY CONDITIONS AND COMPATIBILITY}

We consider problem (1.1) together with the fully nonlinear boundary conditions (1.2).

DEFINITION 2.1: We call $\boldsymbol{\Omega}$ an admissible bounding set for (1.1) if it is a bounded open subset of $[0,1] \times \mathbf{R}^{n}$ with the following properties:

(1) $[0,1] \times\{0\} \subset \boldsymbol{\Omega}$;

(2) $\boldsymbol{\Omega}(x)$ is star shaped with respect to the origin for all $x \in[0,1]$;

(3) the mapping $L(x, y)$ defined by (1.6) is Lipschitz continuous on $[0,1] \times \mathbf{R}^{n}$; 
(4) for each point $(t, u) \in \partial \boldsymbol{\Omega}$ with $t \in[0,1]$ there is neighbourhood $N$ and a continuously differentiable function $r: N \rightarrow \mathbf{R}$ such that

(a) $\Omega \cap N \subset\{(x, y) \in N: r(x, y)<0\}$ and

(b) $r(t, u)=0$,

(c) if $t \in(0,1)$, then $r: N \rightarrow \mathbf{R}$ is twice continuously differentiable and

$r_{f}^{\prime \prime}(t, u, p)=r_{x x}(t, u)+2 r_{x y}^{T}(t, u) p+p^{T} r_{y y}(t, u) p+r_{y}^{T}(t, u) f(t, u, p) \geqslant 0$

for all $p \in \mathbf{R}^{n}$ such that $r^{\prime}(t, u, p)=r_{x}(t, u)+r_{y}^{T}(t, u) p=0$;

(5) there is a continuous vector field $n:[0,1] \times \mathbf{R}^{n} \rightarrow \mathbf{R}^{n}$ such that

$$
\begin{gathered}
n(t, u)^{T} r_{y}(t, u) \geqslant\left|r_{y}(t, u)\right|>0 \text { and } \\
n(t, u)^{T} u \geqslant|u|>0
\end{gathered}
$$

for all $(t, u) \in \partial \boldsymbol{\Omega}$ with $t \in[0,1]$.

Set $\Delta=\Omega(0) \times \Omega(1)$.

REMARK 2.2. It is easy to see that (5) of Definition 2.1 is satisfied if $\partial \boldsymbol{\Omega}$ is smooth and $L$ is uniformly Lipschitz continuous.

In place of (1) of Definition 2.1 we can allow

$$
\{(x, \phi(x)): x \in[0,1]\} \subset \Omega
$$

for some $\phi \in C^{2}[0,1]$, with the appropriate changes in the other assumptions.

It is not difficult to show that Definition 2.1 is equivalent to [11, Definition 3.1].

DEFINITION 2.3: We call $f$ admissible for (1.1) if it satisfies

(1) $f \in C\left([0,1] \times \mathbf{R}^{n} \times \mathbf{R}^{n} ; \mathbf{R}^{n}\right)$,

(2) $|f| \leqslant \phi(|p|)$, where $\int^{\infty} s / \phi(s) d s=\infty$ and

(3) $|f| \leqslant 2 C\left(y^{T} f+|p|^{2}\right)+K$, where $C, K$ are non-negative constants.

Let $G:[0,1] \times[0,1] \rightarrow \mathbf{R}$ be the Green's function for (1.1) together with the homogeneous boundary conditions $A=0=B$ in (1.3). Thus

$$
G(x, t)= \begin{cases}x(1-t), & \text { for } 0 \leqslant x \leqslant t \leqslant 1 \\ (1-x) t, & \text { for } 0 \leqslant t \leqslant x \leqslant 1\end{cases}
$$

Let $w(x, A, B)=A(1-x)+B x$. 
Definition 2.4: We call the vector field $\Psi=\left(\psi^{0}, \psi^{1}\right) \in C\left(\overline{\boldsymbol{\Delta}} ; \mathbf{R}^{2 n}\right)$ strongly inwardly pointing on $\boldsymbol{\Delta}$ if for all $(C, D) \in \overline{\boldsymbol{\Delta}}$

$$
\begin{aligned}
& r^{\prime}\left(0, C, \psi^{0}(C, D)\right)<0, \text { for } C \in \partial \boldsymbol{\Omega}(0) \text { and associated function } r \\
& r^{\prime}\left(1, D, \psi^{1}(C, D)\right)>0, \text { for } D \in \partial \boldsymbol{\Omega}(1) \text { and associated function } r
\end{aligned}
$$

We call $\Psi$ inwardly pointing if the strict inequalities are replaced by weak inequalities.

In what follows where there is a strongly inwardly pointing vector field $\Psi$ on $\bar{\Delta}$ clearly defined from the context $\mathcal{G}$ will denote the vector field defined by

$$
\mathcal{G}(C, D)=g((C, D) ; \Psi(C, D)) \text { for all }(C, D) \in \overline{\boldsymbol{\Delta}} \text {. }
$$

Definition 2.5: Let $g \in C\left(\bar{\Delta} \times \mathbf{R}^{2 n} ; \mathbf{R}^{2 n}\right)$. We say $g$ is strongly compatible with $\Omega$ if

$$
d(\mathcal{G}, \Delta, 0) \neq 0
$$

for any strongly inwardly pointing vector field $\Psi$ on $\overline{\boldsymbol{\Delta}}$ and

$$
g((C, D),(p, q)) \neq 0
$$

for all $(C, D, p, q) \in \overline{\boldsymbol{\Delta}} \times \mathbf{R}^{2 n}$ satisfying either

$$
\begin{array}{ll}
(C, D) \in \partial \boldsymbol{\Omega}(0) \times \boldsymbol{\Omega}(1), & r^{\prime}(0, C, p)<0, \text { or } \\
(C, D) \in \boldsymbol{\Omega}(0) \times \partial \boldsymbol{\Omega}(1), & r^{\prime}(1, D, q)>0 \text { or } \\
(C, D) \in \partial \boldsymbol{\Omega}(0) \times \partial \boldsymbol{\Omega}(1), & r^{\prime}(0, C, p)<0, r^{\prime}(1, D, q)>0 .
\end{array}
$$

We say $g$ is compatible with $\Omega$ if there is a sequence $g_{i} \in C\left(\bar{\Delta} \times \mathbf{R}^{2 n} ; \mathbf{R}^{2 n}\right)$ strongly compatible with $\Omega$ and converging uniformly to $g$ on compact subsets of $\bar{\Delta} \times \mathbf{R}^{2 n}$.

REMARK 2.6. If $g$ is (strongly) compatible with $\boldsymbol{\Omega}$ then the Brouwer degree (2.4) is independent of the strongly inwardly pointing vector field $\Psi$. To see this, for strongly compatible $g$ let $\Psi_{i}, i=1,2$, be two such vector fields. Setting $\Psi(C, D, \theta)=$ $\theta \Psi_{1}(C, D)+(1-\theta) \Psi_{2}(C, D)$ and $\mathcal{H}(C, D, \theta)=g((C, D) ; \Psi(C, D, \theta))$ on $\overline{\boldsymbol{\Delta}} \times[0,1]$, it follows that $\mathcal{H}$ is a homotopy for the Brouwer degree (2.4).

REMARK 2.7. Let $\boldsymbol{\Omega}$ be admissible for (1.1), then there exists a strongly inwardly pointing vector field $\Psi$ on $\boldsymbol{\Omega}$. This can be seen as follows. Since $L(x, y)$ is Lipschitz and $r$ and $\Omega$ satisfy (4) in the definition of admissible, if

$$
\Psi(C, D)=k(-n(0, C), n(1, D))
$$

for all $(C, D) \in \overline{\boldsymbol{\Delta}}$, then $\Psi$ is a strongly inwardly pointing vector field on $\boldsymbol{\Delta}$ for any sufficiently large positive constant $k$. 
REMARK 2.8. Let $\boldsymbol{\Omega}$ be admissible for (1.1). Moreover assume there is a continuously differentiable function $r:[0,1] \times \mathbf{R}^{n} \rightarrow \mathbf{R}$ satisfying (4) and (5) of Definition 2.1 such that $\Omega=\left\{(x, y) \in[0,1] \times \mathbf{R}^{n}: r(x, y)<0\right\}$. Let $\varepsilon>0, \eta>0$ and let $\Psi=\left(\psi^{0}, \psi^{1}\right)$ be defined for all $(C, D) \in \partial \boldsymbol{\Omega}(0) \times \partial \boldsymbol{\Omega}(1)$ by

$$
\Psi(C, D)=\left(\frac{-\left(r_{x}(0, C)+\varepsilon\right) r_{y}(0, C)}{\left|r_{y}(0, C)\right|^{2}}, \frac{\left(r_{x}(1, D)+\eta\right) r_{y}(1, D)}{\left|r_{y}(1, D)\right|^{2}}\right)
$$

Extend $\Psi$ to a continuous $\mathbf{R}^{2 n}$ valued function on $\bar{\Delta}$, then $\Psi$ is a strongly inwardly pointing vector field on $\overline{\boldsymbol{\Delta}}$. Moreover, if (2.5) holds for all inwardly pointing vector fields we may use (2.7) when computing the Brouwer degree (2.4).

Moreover since $\boldsymbol{\Omega}(x)$ is starshaped with respect to 0 and $L(x, y)$ is Lipschitz it follows that $r_{y}(i, y)^{T} y>0$ for all $(i, y) \in \partial \Omega$ for $i=0,1$. Thus, as in Remark 2.7, if

$$
\begin{aligned}
& \Psi(C, D)=k\left(-r_{y}(0, C), r_{y}(1, D)\right), \quad \text { or } \\
& \Psi(C, D)=k(-C, D)
\end{aligned}
$$

for all $(C, D) \in \bar{\Delta}$, then $\Psi$ is a strongly inwardly pointing vector field on $\boldsymbol{\Omega}$ for any sufficiently large positive constant $k$.

Also, in this case (2.5) holds if $\mathcal{G} \neq 0$ for all strongly inwardly pointing vector fields. To see this let $(C, D, p, q) \in \partial \boldsymbol{\Delta} \times \mathbf{R}^{2 n}$ satisfy $C \in \partial \boldsymbol{\Omega}(0)$ and $r^{\prime}(0, C, p)<0$. First assume $D \in \Omega(1)$. Since $r^{\prime}(0, C, p)$ is a continuous function of $E \in \partial \Omega(0)$, there is $\delta>0$ such that $r^{\prime}(0, E, p)>0$ for all $E \in B(C, 2 \delta)$ and $B(D, 2 \delta) \cap \partial \Omega(1)=\emptyset$. Let $\Psi$ be a strongly compatible vector field on $\Delta$ and let

$$
\begin{aligned}
& \psi_{p}^{0}(E, D)= \begin{cases}|E-C| \psi^{0}(E, D) / \delta+(1-|E-C| / \delta) p, & \text { for }(E, D) \in B(C, \delta) \cap \bar{\Delta} \\
\psi^{0}(E, D), & \text { otherwise. }\end{cases} \\
& \psi_{q}^{1}(E, D)= \begin{cases}|E-C| \psi^{1}(E, D) / \delta+(1-|E-C| / \delta) q, & \text { for }(E, D) \in B(C, \delta) \cap \bar{\Delta} \\
\psi^{1}(E, D), & \text { otherwise. }\end{cases}
\end{aligned}
$$

Thus $\left(\psi_{p}^{0}, \psi_{q}^{1}\right)$ is a strongly compatible vector field on $\Delta$ with $\left(\psi_{p}^{0}(C, D), \psi_{q}^{1}(C, D)\right)=$ $(p, q)$. Then $g((C, D),(p, q)) \neq 0$ follows. The case $D \in \partial \boldsymbol{\Omega}(1), C \in \boldsymbol{\Omega}(0)$ and $r^{\prime}(1, D, q)>0$ follows by a similar argument. The case $C \in \partial \boldsymbol{\Omega}(0), D \in \partial \boldsymbol{\Omega}(1)$ $r^{\prime}(0, C, p)<0, r^{\prime}(1, D, q)>0$ follows by the above choice of $\left(\psi_{p}^{0}, \psi_{q}^{1}\right)$ where $\delta>0$ is chosen such shat $r^{\prime}(0, E, p)<0, r^{\prime}(1, F, q)>0$ for all $E \in B(C, 2 \delta)$ and $F \in B(D, 2 \delta)$.

In the case $\Omega$ is an admissible bounding set given by $r \in C^{1}\left([0,1] \times \mathbf{R}^{n} ; \mathbf{R}\right)$ as in Remark 2.8, the compatibility conditions for the Picard, Neumann, and periodic boundary conditions become

$$
\begin{gathered}
A \in \overline{\boldsymbol{\Omega}}(0), \quad B \in \overline{\mathbf{\Omega}}(1), \\
r(0, C)=0 \Rightarrow r^{\prime}(0, C, A) \leqslant 0, r(1, D)=0 \Rightarrow r^{\prime}(1, D, B) \geqslant 0,
\end{gathered}
$$


and

$$
\begin{aligned}
\boldsymbol{\Omega}(0) & =\boldsymbol{\Omega}(1) \\
r_{y}(0, C) & =t(C) r_{y}(1, C) \\
r_{x}(0, C) & \geqslant t(C) r_{x}(1, C), \quad t(C)>0, \quad C \in \partial \boldsymbol{\Omega}(0)=\partial \boldsymbol{\Omega}(1),
\end{aligned}
$$

respectively.

We prove this in the case of periodic boundary conditions. First we need the following lemma.

LEMMA 2.9. Let

$$
\mathcal{J}(C, D)=(C-D, J(C, D)) \text { for all }(C, D) \in \overline{\boldsymbol{\Delta}}
$$

where $J \in C\left(\overline{\mathbf{\Delta}} ; \mathbf{R}^{n}\right)$. If $Q=\boldsymbol{\Omega}(0) \cap \boldsymbol{\Omega}(1)$ and $j \in C\left(\bar{Q} ; \mathbf{R}^{n}\right)$ is defined by $j(P)=$ $J(P, P)$ for all $P \in \bar{Q}$, then $d(\mathcal{J}, \Delta, 0)=d(j, Q, 0)$.

Proof: Let $K=\{\bar{\Omega}(0) \backslash Q\} \times\{\bar{\Omega}(1) \backslash Q\}$. Then $K$ is closed and $\mathcal{J} \neq 0$ for all $(C, D) \in K$. Since $Q \times Q=\Delta \backslash K$, by the excision property of degree

$$
d(\mathcal{J}, \Delta, 0)=d(\mathcal{J}, Q \times Q, 0)
$$

Setting $(W, Z)=\Gamma(C, D)=(C-D, C+D)$,

$$
\mathcal{J} \circ \Gamma^{-1}(W, Z)=(W, j((W+Z) / 2,(Z-W) / 2))
$$

then

$$
d(\mathcal{J}, Q \times Q, 0)=d\left(\mathcal{J} \circ \Gamma^{-1}, \Gamma(Q \times Q), 0\right)
$$

and the result follows by the reduction property of degree.

LEMMA 2.10. Let $\Omega$ be an admissible bounding set given by $r \in C^{1}\left([0,1] \times \mathbf{R}^{n}\right.$; $\mathbf{R}$ ) as in Remark 2.8. Then periodic boundary conditions are (strongly) compatible if and only if (2.10) holds.

PROOF: Let $\Psi$ be a strongly inwardly pointing vector field on $\overline{\boldsymbol{\Delta}}$.

Assume that $(2.10)$ is satisfied, let $g=\left(g^{0}, g^{1}\right)$ be given by $(1.5)$ and $(C, D) \in \partial \Delta$. If $\mathcal{G}(C, D)=0$ then $C=D \in \partial \boldsymbol{\Omega}(0)=\partial \boldsymbol{\Omega}(1)$ and $\psi^{0}(C, D)=\psi^{1}(C, D)$ so that $0>r^{\prime}\left(0, C, \psi^{0}(C, D)\right) \geqslant t(C) r^{\prime}\left(1, D, \psi^{1}(C, D)\right)>0$, a contradiction. Thus $\mathcal{G} \neq 0$ on $\partial \Delta$. Now $\mathcal{H}(C, D, \theta)=(1-2 \theta) \mathcal{G}(C, D)+2 \theta\left(\mathcal{G}^{0}(C, D), D\right)$, for $\theta \in[0,1 / 2]$ and $\mathcal{H}(C, D, \theta)=(2-2 \theta)\left(\mathcal{G}^{0}(C, D), D\right)+(2 \theta-1)(C, D)$, for $\theta \in[1 / 2,1]$. Since $\mathcal{H}$ is a homotopy for Brouwer degree $d(\mathcal{G}(\cdot), \Delta, 0)=d(\mathcal{H}(\cdot, 0), \Delta, 0)=d(\mathcal{H}(\cdot, 1), \Delta, 0)=1 \neq$ 0 . Thus $G$ is strongly compatible and hence compatible. 
Assume now that $g$ is given by (1.5) and that $g$ is strongly compatible with $\Omega$. We show that $(2.10)$ is satisfied. First we show that $\boldsymbol{\Omega}(0)=\boldsymbol{\Omega}(1)$. Assume that $\Omega(0) \neq \Omega(1)$. Let $\Psi$ be a strongly inwardly pointing vector field on $\Delta$. Thus

$$
\mathcal{G}(C, D)=\left(C-D, \psi^{0}(C, D)-\psi^{1}(C, D)\right) \neq 0 \text { for all }(C, D) \in \partial \Delta
$$

Setting $Q=\boldsymbol{\Omega}(0) \cap \boldsymbol{\Omega}(1)$ and $j(P)=\psi^{0}(P, P)-\psi^{1}(P, P)$ for all $P \in \bar{Q}$, by Lemma 2.9 we have $d(\mathcal{G}, \Delta, 0)=d(j, Q, 0) \neq 0$. Without loss of generality we may assume that $\boldsymbol{\Omega}(0) \neq Q$. We construct a strongly compatible vector field $\Psi$ with $d(j, Q, 0)=0$, a contradiction, and then $\boldsymbol{\Omega}(0)=\boldsymbol{\Omega}(1)$. Without loss of generality we may assume that $e_{1}=(1,0, \ldots, 0) \in \Omega(0) \cap \partial Q$, where $\partial Q$ is the boundary of $Q$. Since $\Omega(0)$ is an open set we may choose $\delta_{0} \in\left(0, \delta_{0} / 2\right)$ such that $B\left(e_{1}, \delta_{0}\right) \subset \boldsymbol{\Omega}(0)$. Set $\lambda=1-\delta_{0} / 2$. By Remark 2.8 we may choose $k>0$ such that $(-k C, k D)$ is strongly inwardly pointing on $\Delta$. Let $\theta(P)$ be the angle between $P$ and $e_{1}$ and $\mathcal{K}_{\delta}$ be the cone

$$
\mathcal{K}_{\delta}=\left\{P \in \mathbf{R}^{n}: \theta(P)<\sin ^{-1}(\delta)\right\}
$$

Let $S_{\delta}=\mathcal{K}_{\delta} \cap\left\{P \in \mathbf{R}^{n}:|P|>\lambda\right\} \cap Q$ where $\delta>0$ is chosen sufficiently small that $|P|>(4+\lambda) / 5$ for all $P \in \partial Q \cap \bar{S}_{\delta}$. Thus $C_{\lambda}=(1+\lambda) e_{1} / 2 \in S_{\delta}$. Set

$$
\gamma_{t}(C)= \begin{cases}1, & \text { if }\left|C-e_{1}\right| \leqslant \delta_{0} / 2 \\ 0, & \text { if }\left|C-e_{1}\right| \geqslant \delta_{0} \\ 1-2\left(\left|C-e_{1}\right|-\delta_{0} / 2\right) / \delta_{0}, & \text { otherwise, }\end{cases}
$$

and $\eta_{t}(C)=\gamma_{t}(C)\left[\left(C-C_{\lambda}\right)-C\left(C-C_{\lambda}\right) \cdot C /|C|^{2}\right]$. Let $\eta_{r}(C)=\gamma_{r}(C) C$ where $\gamma_{r}$ is continuous and satisfies

$$
\gamma_{r}(C)= \begin{cases}3-\sin (\theta(C)) / \delta, & \text { if } \theta \leqslant \sin ^{-1}(\delta),|C| \geqslant(3+\lambda) / 4 \\ 0, & \text { if } \theta \geqslant \sin ^{-1}(3 \delta) \text { or }|C| \leqslant(1+\lambda) / 2\end{cases}
$$

Set $\Psi(C, D)=\left(-k C+(-1)^{n+1} \varepsilon \eta_{t}(C)+\eta_{r}(C), k D\right)$ where $\varepsilon>0$ is chosen sufficiently small that $\Psi$ is strongly inwardly pointing on $\Delta$.

It follows from the additivity and excision properties of degree that

$$
d(j, Q, 0)=d\left(j, Q \backslash \bar{S}_{\delta}, 0\right)+d\left(j, S_{\delta}, 0\right)
$$

Also $d\left(j, Q \backslash \bar{S}_{\delta}, 0\right)=(-1)^{n}$ since $0 \in Q \backslash \bar{S}_{\delta}$ and $j$ is homotopic to $-I$ there. Also $d\left(j, S_{\delta}, 0\right)=(-1)^{n+1}$ since $(2+\lambda) e_{1} / 3 \in S_{\delta}$, and $j(P)$ is homotopic to $I^{-}-(2+\lambda) e_{1} / 3$ if $n$ is even while $j(P)$ is homotopic to $I-(2+\lambda) e_{1} / 3$ if $n$ is odd. Thus $d(j, Q, 0)=0$, a contradiction, and $\boldsymbol{\Omega}(0)=\boldsymbol{\Omega}(1)$. Thus $r_{y}(0, C)=\boldsymbol{t}(C) r_{y}(1, C)$ for $C \in \partial \Omega(0)=\partial \Omega(1)$, where $t=t(C)>0$ is continuous. 
We show that $r_{x}(0, C) \geqslant t(C) r_{x}(1, C), \quad t(C)>0$, for all $C \in \partial \boldsymbol{\Omega}(0)=\partial \boldsymbol{\Omega}(1)$. Assume the result is false. Without loss of generality we may assume that $e_{1} \in \partial \boldsymbol{\Omega}(0)=$ $\partial \boldsymbol{\Omega}(1)$ and $r_{x}\left(0, e_{1}\right)<t\left(e_{1}\right) r_{x}\left(1, e_{1}\right)$. Again we set $Q=\boldsymbol{\Omega}(0) \cap \boldsymbol{\Omega}(1)=\boldsymbol{\Omega}(0)=\boldsymbol{\Omega}(1)$. The result follows by an argument similar to the previous one in view of the following.

The key to the previous argument was constructing $\Psi$ on $\partial Q$ such that $j(C)=$ $\kappa(C) C+(-1)^{n+1} \varepsilon \eta_{t}(C)$, where $\varepsilon>0$ is sufficiently small and $\kappa(C)$ is continuous and satisfies

$$
\begin{array}{ll}
\kappa(C)<0, & \text { if } \theta(C)>\sin ^{-1}(\delta) \text { and } \\
\kappa(C)>0, & \text { if } \theta(C)<\sin ^{-1}(\delta) .
\end{array}
$$

Since $r_{x}\left(0, e_{1}\right)<t\left(e_{1}\right) r_{x}\left(1, e_{1}\right)$ and $t$ and $r_{x}$ are continuous we may choose $\delta_{0}>0$ and $l$ such that for all $C \in \partial Q \cap \bar{B}\left(e_{1}, \delta_{0}\right)$,

$$
\begin{aligned}
& r_{x}(0, C)+r_{y}(0, C)\left(l+\delta_{0}\right) C<0, \text { and } \\
& r_{x}(1, C)+r_{y}(1, C)\left(l-\delta_{0}\right) C>0 .
\end{aligned}
$$

As in Remark 2.8 we may choose $k>0$ such that

$$
\begin{aligned}
& r_{x}(0, C)-r_{y}(0, C) k C<0, \text { and } \\
& r_{x}(1, C)+r_{y}(1, C) k C>0,
\end{aligned}
$$

for all $C \in \partial Q$.

Set $\psi_{\delta}^{1}(C, D)=\omega_{1, \delta}(D) D$, where $\omega_{1, \delta}$ is defined by

$$
\omega_{1, \delta}(D)= \begin{cases}l-\delta+\sin \theta(D), & \text { if } 0 \leqslant \theta \leqslant \sin ^{-1}(\delta) \\ l(2 \delta-\sin \theta(D)) / \delta+k(\sin \theta(D)-\delta) / \delta, & \text { if } \sin ^{-1}(\delta) \leqslant \theta \leqslant \sin ^{-1}(2 \delta) \\ k, & \text { otherwise, }\end{cases}
$$

Set $\psi_{\delta}^{0}(C, D)=\omega_{0, \delta}(C) C+(-1)^{n+1} \varepsilon \eta_{t}(C)$, where $\omega_{0, \delta}$ is defined by

$$
\omega_{0, \delta}(C)= \begin{cases}l, & \text { if } 0 \leqslant \theta \leqslant \sin ^{-1}(\delta) \\ l(2 \delta-\sin \theta(C)) / \delta-k(\sin \theta(C)-\delta) / \delta, & \text { if } \sin ^{-1}(\delta) \leqslant \theta \leqslant \sin ^{-1}(2 \delta) \\ -k, & \text { otherwise. }\end{cases}
$$

Set $\Psi_{\delta}(C, D)=\left(\psi_{\delta}^{0}(C, D), \psi_{\delta}^{1}(C, D)\right)$ for all $(C, D) \in \partial \Delta$. It follows that $\Psi$ is the restriction to $\partial \boldsymbol{\Delta}$ of a strongly inwardly pointing vector field on $\boldsymbol{\Delta}$ for $\varepsilon>0$ sufficiently small and $\delta \in\left(0, \delta_{0} / 2\right)$.

By choosing $\delta>0$ small enough we may extend $\Psi_{\delta}$ to $\bar{\Delta}$ as in the previous argument, to obtain the contradiction that $d(j, Q, 0)=0$. Thus $r_{x}(0, C) \geqslant t(C) r_{x}(1, C)$ for all $C \in \partial \boldsymbol{\Omega}(0)$. Thus $r$ satisfies (2.10) as required.

The proof that the Picard, respectively Neumann, boundary conditions are compatible if and only if (2.8), respectively (2.9), is satisfied is simpler, follows similar lines and hence is omitted. 
REMARK 2.12. Let $n=1$ and suppose there exist lower and upper solutions $\alpha$ and $\beta, \alpha(x)<\beta(x)$ for all $x$ in $[0,1]$. Set $\boldsymbol{\Omega}=\{(x, y): \alpha(x)<y<\beta(x), x \in[0,1]\}$, then $\boldsymbol{\Omega}$ is an admissible bounding set for (1.1) since we may set

$$
r(x, y)=(y-\alpha(x))(y-\beta(x))
$$

Then conditions (2.8), (2.9), and (2.10) become

$$
\begin{gathered}
\alpha(0) \leqslant A \leqslant \beta(0), \quad \alpha(1) \leqslant B \leqslant \beta(1), \\
\alpha^{\prime}(0) \geqslant A \geqslant \beta^{\prime}(0), \quad \alpha^{\prime}(1) \leqslant B \leqslant \beta^{\prime}(1)
\end{gathered}
$$

and

$$
\begin{array}{cc}
\alpha(0)=\alpha(1), & \beta(0)=\beta(1), \\
\alpha^{\prime}(0) \geqslant \alpha^{\prime}(1), & \beta^{\prime}(0) \leqslant \beta^{\prime}(1),
\end{array}
$$

respectively.

REMARK 2.13. It is not difficult to construct examples to show that some compatiblity assumptions of this kind are necessary to guarantee existence. In the following two examples $g$ is not strongly compatible since condition (2.4) fails. In the first there is a solution $y$ of (1.1) and (1.2) satisfying $(x, y(x)) \in \overline{\mathbf{\Omega}}=\left\{(x, y) \in \mathbf{R}^{2}: x \in\right.$ $[0,1], \alpha(x) \leqslant y(x) \leqslant \beta(x)\}$ where $\alpha$ and $\beta$ are upper and lower solutions for (1.1) given below (see [10]). In the second example there are no such solutions. We choose $R>0$ and $g \in C\left([-R, R]^{2} \times \mathbf{R}^{2} ; \mathbf{R}^{2}\right)$ such that $G((C, D) ;(P, Q))=G((C, D) ;(S, T))$ for all $((C, D) ;(P, Q)),((C, D) ;(S, T)) \in[-R, R]^{2} \times \mathbf{R}^{2}, \mathcal{G} \neq 0$ on $\partial(-R, R)^{2}$ and $d\left(\mathcal{G},(-R, R)^{2}, 0\right)=0 ; \mathcal{G}$ is independent of the choice of strongly inwardly pointing vector field $\Psi$. Let $f$ be identically zero and $-\alpha=R=\beta$. For this choice of $f, \alpha$, and $\beta$ it is easy to see that $\boldsymbol{\Omega}$ is an admissible bounding set for (1.1).

For the first example we choose $G$ so that $g((C, D) ;(P, Q))=0$, for some $(C, D) \in$ $(-R, R)^{2}$. For the second example we choose $G$ so that $g \neq 0$ for any $(C, D) \in$ $(-R, R)^{2}$.

\section{The MAIN RESUlT}

THEOREM 3.1. Let $f$ be admissible, $\Omega$ be an admissible bounding set for (1.1) and let $g \in C\left(\overline{\boldsymbol{\Delta}} \times \mathbf{R}^{2 n} ; \mathbf{R}^{2 n}\right)$ be compatible with $\boldsymbol{\Omega}$. Then problem (1.1) and (1.2) has a solution $y$ with $(x, y) \in \bar{\Omega}$, for all $x \in[0,1]$.

ProOF: We assume first that $r_{f}^{\prime \prime}>0$ when $r=0=r^{\prime}$. We use Schauder degree theory and need the following family of functions to construct a homotopy. 
Choose $R>0$ and $\varepsilon \in(0,1)$ such that $B_{2 \varepsilon} \subseteq \Omega(x) \subseteq B_{R}$ for all $x \in[0,1]$. Let $h \in C\left(\mathbf{R}^{n} ;[0,1]\right)$ satisfy

$$
\begin{gathered}
h(y)= \begin{cases}0, & \text { if }|y|<\varepsilon \\
1, & \text { if }|y|>2 \varepsilon\end{cases} \\
f_{\lambda}(x, y, p)=\lambda f(x, y, p)+(1-\lambda) h(y)|f| n(x, y) \text { and } \\
g_{\lambda}(x, y, p)=L(x, y) f_{\lambda}(x, y / L(x, y), p / L(x, y))
\end{gathered}
$$

where $L$ is given in (1.6). Thus $g_{\lambda}$ is continuous, $f_{\lambda}=g_{\lambda}$ in $\Omega \times \mathbf{R}^{n}$ and it suffices to find a solution $y$ of (3.2) and (1.2) with $\lambda=1$ and $(x, y) \in \bar{\Omega}$. Let $\Pi=\{y \in$ $C^{\mathbf{1}}\left([0,1] ; \mathbf{R}^{n}\right):|y|<R$ and $\left.\left|y^{\prime}\right|<M\right\}$, where $M$ is chosen below. Let $\boldsymbol{\Sigma}=\boldsymbol{\Pi} \times \boldsymbol{\Delta}$.

For $y \in C^{1}\left([0,1] ; \mathbf{R}^{n}\right)$, we set

$$
T\left(g_{\lambda}(y)\right)(x)=-\int_{0}^{1} G(x, t) g_{\lambda}\left(t, y(t), y^{\prime}(t)\right) d t
$$

If $(y, C, D) \in \bar{\Sigma}$ is a solution of

$$
\left(y(x)-T\left(g_{1}(y)\right)\right)-w(x, C, D), g\left((C, D) ;\left(y^{\prime}(0), y^{\prime}(1)\right)\right)=0
$$

we show that $(x, y) \in \overline{\boldsymbol{\Omega}}$. Thus, by the definition of $T$ and $g_{\lambda}$, problem (1.1) and (1.2) has a solution $y$ with $(x, y) \in \bar{\Omega}$ if and only if $(y, C, D)$ is a solution of (3.3) in $\bar{\Sigma}$. We choose $M$ as follows. By continuity, $L \leqslant l$ on $[0,1] \times \bar{B}_{R}$, for some $l \geqslant 1$. Set

$$
c=\sup \{|n(x, y)|:(x, y) \in \mathbf{\Omega}\}
$$

Thus $\left|g_{\lambda}\right| \leqslant c \phi(|p|)$ and $\left|g_{\lambda}\right| \leqslant 2(C+c / \varepsilon)\left(y^{T} f+|p|^{2}\right)+K l^{2}$, for all $(x, y) \in \boldsymbol{\Omega}$. By Hartman $[9$, Lemma 5.2, p.429] there is $M$ such that solutions $y$ of

$$
y^{\prime \prime}=g_{\lambda}\left(x, y, y^{\prime}\right) \in[0,1]
$$

and (1.2) with $(x, y) \in \bar{\Omega}$ satisfy $\left|y^{\prime}\right|<M$. To show that (3.3) has a solution we use Schauder degree theory. Define $H_{i}:[0,1] \times \bar{\Sigma} \rightarrow C^{1}\left([0,1] ; \mathbf{R}^{n}\right) \times \mathbf{R}^{n}$ for $i=1,2,3$, by

$$
\begin{aligned}
& H_{1}(\lambda,(y, C, D))(x)=\left(y(x)-T\left(g_{1}(y)\right)(x)-w(x, C, D), \mathcal{S}(y, C, D, \lambda)\right) \\
& H_{2}(\lambda,(y, C, D))(x)=\left(y(x)-T\left(g_{\lambda}(y)\right)(x)-w(x, C, D), \mathcal{G}(C, D)\right) \text { and } \\
& H_{3}(\lambda,(y, C, D))(x)=\left(y(x)-\lambda\left(T\left(g_{0}(y)\right)(x)-w(x, C, D)\right), \mathcal{G}(C, D)\right)
\end{aligned}
$$

where $\mathcal{S}(y, C, D, \lambda)=g\left((C, D) ; \lambda\left(y^{\prime}(0), y^{\prime}(1)\right)+(1-\lambda) \Psi(C, D)\right)$ 
We show that either there is a solution to our problem or the above functions $H_{i}$ define homotopies.

Suppose $H_{1}(\lambda,(y, C, D))=0$ has a solution $(y, C, D) \in \partial \boldsymbol{\Sigma}$. By the choice of $M,\left|y^{\prime}\right|<M$. Suppose $y \in \partial \Pi$. By the choice of $R$ there is $t \in(0,1)$ such that $|y(t)|=R$ and $L(t)>1$. As $L(0)=1=L(1)$ and $L$ is continuous, there is $t_{0} \in$ $(0,1)$ such that $L$ has a maximum $l_{0} \leqslant l$ say, at $t_{0}$. Let $z(x)=y(x) / l_{0}$. Thus $(x, z) \in \bar{\Omega}$ for all $x \in[0,1]$ and $\left(t_{0}, y\left(t_{0}\right)\right) \in \partial \boldsymbol{\Omega}$. Thus $r\left(t_{0}, z\right)=0, r^{\prime}\left(t_{0}, z, z^{\prime}\right)=0$ and $r_{f}^{\prime \prime}\left(t_{0}, z, z^{\prime}\right)>0$, a contradiction, and $y \notin \partial \Pi$. Since $y(0)=C, y(1)=D$ and $(C, D) \in \overline{\boldsymbol{\Delta}}$ it follows that $(x, y) \in \overline{\boldsymbol{\Omega}}$ for all $x \in[0,1]$. Suppose $y(0)=C \in \partial \boldsymbol{\Omega}(0)$. If $\lambda=1$, then $(y, C, D)$ is a solution to our problem, as required. If $0 \leqslant \lambda<1$, then $r(0, C)=0$ and $r^{\prime}\left(0, C, y^{\prime}\right) \leqslant 0$ so $r^{\prime}\left(0, C, \lambda y^{\prime}(0)+(1-\lambda) \psi^{0}(C, D)\right)<0$, and thus $g\left((C, D) ; \lambda\left(y^{\prime}(0), y^{\prime}(1)\right)+(1-\lambda) \Psi(C, D)\right) \neq 0$, a contradiction. A similar argument shows that $y(1)=D \notin \partial \boldsymbol{\Omega}(1)$. Thus $H_{1}(\lambda,(y, C, D)) \neq 0$ for any $(y, C, D) \in \partial \boldsymbol{\Sigma}$.

Suppose $H_{2}(\lambda,(y, C, D))=0$ has a solution $(y, C, D) \in \partial \mathbf{\Sigma}$. Suppose $y \in \partial \Pi$. Since $\left|y^{\prime}\right|<M,|y(t)|=R$ for some $t \in(0,1)$. As above $L$ has a maximum $l_{0}>1$ at $t_{0} \in(0,1)$ and by setting $z(x)=y(x) / l_{0}$ we again get a contradiction, $r\left(t_{0}, z\right)=$ $0, r^{\prime}\left(t_{0}, z, z^{\prime}\right)=0$ and $r_{g_{\lambda}}^{\prime \prime}\left(t_{0}, z, z^{\prime}\right)>0$, since $r_{y}(0, z)\left(n\left(t_{0}, z\right) h(z)\left|f\left(t_{0}, z, z^{\prime}\right)\right|-\right.$ $\left.f\left(t_{0}, z, z^{\prime}\right)\right) \geqslant 0$, by condition (5) of the definition of admissibility of $\boldsymbol{\Omega}$. Now $\mathcal{G}(C, D) \neq 0$ on $\partial \boldsymbol{\Delta}$ so $H_{2} \neq 0$ on $\partial \boldsymbol{\Sigma}$.

Suppose $H_{3}(\lambda,(y, C, D))=0$ has a solution $(y, C, D) \in \partial \boldsymbol{\Sigma}$. By the above arguments it suffices to show that there is no $t \in(0,1)$ such that $|y(t)|=R$. If such a $t$ exists then $\lambda>0, y(t)^{T} y^{\prime}(t)=0$ and $y^{\prime}(t)^{2}+y^{\prime \prime}(t)^{T} y(t) \geqslant y^{\prime}(t)^{2}+\lambda l|f||y(t)|>0$, since $n(t, y / l)^{T} y \geqslant|y|$, where $l=L(t, y(t))>1$. This is a contradiction, so $H_{3} \neq 0$ on $\partial \boldsymbol{\Sigma}$.

By the homotopy invariance of Schauder degree

$$
d\left(H_{i}(\lambda, \cdot), \boldsymbol{\Sigma}, 0\right)=\text { constant }
$$

for all $\lambda \in[0,1]$ and $i=1,2,3$. In particular,

$$
\begin{aligned}
d\left(H_{1}(1, \cdot), \boldsymbol{\Sigma}, 0\right) & =d\left(H_{2}(\lambda, \cdot), \boldsymbol{\Sigma}, 0\right) \\
& =d\left(H_{3}(0, \cdot), \boldsymbol{\Sigma}, 0\right)=1 .
\end{aligned}
$$

Thus there is solution in $\boldsymbol{\Sigma}$ of $H_{1}(1,(y, C, D))=\mathbf{0}$, and by the above arguments $y$ is the required solution of (1.1) and (1.2).

If $r_{f}{ }_{f} \geqslant 0$ when $r=0=r^{\prime}$, consider the sequence of problems where $f$ is replaced by $f+y / n$. By the above argument there exists a sequence of solutions $y_{n}$ of these which will have a subsequence, convergent to the required solution.

Theorem 3.1 includes some well known results for the Picard, the periodic and the Neumann boundary conditions as special cases; see for example, Bebernes [2], Bebernes and Schmitt [1], Habets and Schmitt [5], Gaines and Mawhin [3], Knobloch [6], 
Knobloch and Schmitt [7], Lan [8] and Mawhin [9]. In particular [11, Theorems 3.2, 3.4 and 3.6] follow immediately from Theorem 3.1; see [11] for more detailed comments.

\section{REFERENCES}

[1] J.W. Bebernes and K. Schmitt, 'Periodic boundary value problems for systems of second order ordinary differential equations', J. Differential Equations 13 (1973), 32-47.

[2] J.W. Bebernes, 'A simple alternative problem for finding periodic solutions of second order ordinary differential systems', Proc. Amer. Math. Soc. 42 (1974), 121-127.

[3] R.E. Gaines and J.L. Mawhin, Coincidence degree and nonlinear differential equations, Lecture Notes in Mathematics 568 (Springer-Verlag, Berlin, Heidelberg, New York, 1977).

[4] A. Granas, R.B. Guenther and J.W. Lee, Nonlinear boundary value problems for ordinary differential equations (Dissertationes Mathematicae 244, Warsaw, 1985).

[5] P. Habets and K. Schmitt, 'Nonlinear boundary value problems for systems of differential equations', Arch. Math. 40 (1983), 441-446.

[6] H.-W. Knobloch, 'On the existence of periodic solutions for second order vector differential equations', J. Differential Equations 9 (1971), 67-85.

[7] H.-W. Knobloch and K. Schmitt, 'Non-linear boundary value problems for systems of differential equations', Proc. Roy. Soc. Edinburgh 78 (1977), 139-159.

[8] Chin-Chin Lan, 'Boundary value problems for second and third order differential equations', J. Differential Equations 18 (1975), 258-274.

[9] J.L. Mawhin, Topological degree methods in nonlinear boundary value problems, Regional Conference Series in Mathematics 40 (American Mathematical Society, Providence R.I., 1979).

[10] H.B. Thompson, 'Second order ordinary differential equations with fully nonlinear two point boundary conditions', Pacific J. Math. 172 (1996), 255-277.

[11] H.B. Thompson, 'Existence of periodic solutions for first and second order systems of ordinary differential equations', Bull. Austral. Math. Soc. 54 (1996), 397-410.

Department of Mathematics

The University of Queensland

Queensland 4072

Australia 